\title{
Offline signature authenticity verification through unambiguously connected skeleton segments
}

\author{
Jugurta Montalvão, Luiz Miranda, and Jânio Canuto
}

\begin{abstract}
A method for offline signature verification is presented in this paper. It is based on the segmentation of the signature skeleton (through standard image skeletonization) into unambiguous sequences of points, or unambiguously connected skeleton segments corresponding to vectorial representations of signature portions. These segments are assumed to be the fundamental carriers of useful information for authenticity verification, and are compactly encoded as sets of 9 scalars (4 sampled coordinates and 1 length measure). Thus signature authenticity is inferred through Euclidean distance based comparisons between pairs of such compact representations. The average performance of this method is evaluated through experiments with pseudooffline versions of signatures from the MCYT-100 database, and true ones from the MCYT-75 database. For comparison purposes, three other approaches are applied to the same set of signatures, namely: (1) a straightforward approach based on Dynamic Time Warping distances between segments, (2) a published method also based on DTW, and (3) the average (nonspecialist) human performance based on Mean Opinion Scores (MOS) under equivalent experimental protocol. Best results close to (3) - are obtained when signature skeleton details are discarded through its sub-sampling to 4 points per segment, and scores from segment shape and length are fused.
\end{abstract}

Index Terms-Offline signature verification, Skeletonization, Mean Opinion Score (MOS), Biometrics.

\section{INTRODUCTION}

$\mathbf{H}$ ANDWRITTEN signature is a form of personal identification widely accepted, both socially and legally, and it has been used for centuries to authenticate documents such as bank checks, letters, contracts and many other that require proof of authorship. By signing, a person may provide unique information regarding the way she or he converts gesture intentions into spontaneous hand movement. Writing speed, traversed path, pen tilt, pressure applied, all these data are articulated to result in a static figure on signed documents [22].

Signature analysis can be divided in two categories: offline and online. In the offline mode, either signatures are available through the traditional wet ink method (such as in paper documents), or they are available in scanned form, through optical devices, such as scanners and digital cameras. In both cases, all available data corresponds to static signature images.

In the online mode, a person uses a digitizing device (e.g. digitizing tablets or touchsceen devices) to directly record signals from the hand movement. This provides much more information than a static image, for the digitizing device typically can record several complementary signals, such as

J. Montalvão, Luiz Miranda, and Jânio Canuto are with the Federal University of Sergipe (UFS), São Cristóvão, Brazil e-mail: jmontalvao(at)ufs.br.

Digital Object Identifier: 10.14209/jcis.2018.33 the path travelled by the pen tip, as well as its instantaneous speed, applied pressure and pen tilt. This approach is the one that dominates research on signature verification now, due to the worldwide spreading of affordable acquisition devices [25].

However, the offline approach still has some attractive aspects. For instance, even today, many contracts and credit card authorization are performed through traditional signatures on paper. Indeed, in many practical situations, for economical or practical reasons, wet ink signatures are yet useful biometric signals. And even in the unlikely scenario of a complete substitution of wet ink signatures by electronically acquired ones, at least the task of signature verification from ancient ink on paper documents should remain a relevant topic, due to the large amount of old signed documents, whose authenticity are potentially waiting to be verified [26].

To give some fundamental definitions and jargon, we assume that a signature verification is a process that determines whether a tested signature was produced by a target individual, from which at least one genuine signature is available. If under some chosen criteria the tested signature is similar to the genuine references, below a pre-established similarity threshold, it is labelled true, or a genuine signature. Otherwise, the signature is labelled false, or a forgery. Moreover, Coetzer et al. [4] classify forgeries as

- Random forgery: The forger does not know the author's name neither the original signature. Thus the false signature is completely random.

- Simple forgery: The forger knows the author's name, but she/he does not have access to the original signature.

- Skilled forgery: The forger has access to samples of genuine signatures, and also knows the name of the author. It can also be divided into two classes: Amateur and Professional. The Professional Skilled Forgery is produced by a person with professional expertise in handwriting analysis, being able to produce a higher quality forgery than the Amateur.

In general, the offline signature verification process can be divided into four steps [1]: Acquisition, Preprocessing, Feature Extraction and Comparison. In the Preprocessing step, image quality is improved and pixels are transformed to reduce the computational burden of the subsequent steps. Examples of techniques applied in this step are: thinning, color conversion, noise reduction, smoothing, morphological operations and resizing. For instance, Shah et al. [28] cropped images to exclude redundant white regions. The Feature Extraction step is where most works propose innovations. According to Batista et al. [2], an ideal feature extraction technique extracts a minimal 
feature set that maximizes interpersonal variability amongst signature samples from various subjects, whereas it minimizes intrapersonal variability amongst samples belonging to the same subject. Lee and Pan [20] divide the features into three classes: Global Features, Local Features and Geometrical Features.

Typical features extracted from offline signatures are marginal projections. Shanker and Rajagopalan [29] extract vertical projection of bitmaps corresponding to signatures, thus yielding profiles which are compared through Dynamic Time Warping (DTW). Likewise, Coetzer et al. [4] pushes a bit further the same idea, by using many marginal projections of the same signature, over different angles, what they call Discrete Radon Transform, whose behaviour is modelled with a Hidden Markov Model. Nguyen et al. [23] also use similar projections. Indeed, they use two techniques for global features extraction: the first is derived from the total energy a writer uses to create a signature, whereas the second technique employs information from the vertical and horizontal projections of a signature, focusing on the proportion of the distance between key strokes in the image, and the height/width of the signature.

Although marginal projections are more commonly used in literature, other approaches to feature extraction may also rely upon texture-based methods, interest-point-based methods, learned representations (see [17], [25] and references therein). Moreover, considering features more closely related to pen trajectory recovery, a particularly relevant work was done in [27] and [30], where the full online trajectory is estimated. In general, regarding trajectory recovery, image skeletonization is frequently used to map offline signatures into sets of points, similar to online representations, which is appealing because online verification techniques may be deployed, such as the use of DTW to compare segments of points from different signatures, as it is not the focus of this work to discuss the effectiveness of skeletonization methods, more on that can be seen in [8], [9], [10], [11]. Indeed, this straightforward approach corresponds to the baseline method implemented in this paper, as explained in Section III.

Also inspired by online approaches, a new method is proposed, through the compact codification of segments of skeletonized offline signatures, as explained in Section IV. These skeletonized segments are the basic aspect of this work, and they are presented in Section II, and further detailed in Section IV. Public databases and experimetal protocols used in this work are explained in Section V.

Experimental results are presented and briefly discussed in Section VI, including a comparison to human baseline performance obtained for the same dataset and protocol, in the spirit of works such as [5] and [21]. Finally, results are compared to state-of-the-art approaches, in Section VII, before some concluding remarks presented in Section VIII.

\section{UNAMBIGUOUSLY CONNECTED SKELETON SEGMENTS}

Raw online signature signals are frequently represented by two vectors of samples: a sequence of regularly sampled horizontal positions, $x_{O N L I N E}(n)$, and another sequence of corresponding vertical positions, $y_{O N L I N E}(n)$, where $n$ stands for sample counter through time. As compared to offline representation, signature verification through signals $x_{O N L I N E}(n)$ and $y_{O N L I N E}(n)$ is significantly better.

Although we know that velocity information may not be completely recovered from offline representations, we address the offline signature verification problem by first recovering horizontal and vertical signals, which may be regarded as pseudo-versions of $x_{O N L I N E}(n)$ and $y_{O N L I N E}(n)$. It is known from works like [12], [13], [14], [16] that skeletons are a good representation for offline signatures that have ribbon like shapes, for this reason the recovering process is done through standard skeletonization, as described in [8], and was chosen for being simple enough and yet able to satisfactorily process the ribbon like shapes of offline signatures. Other more complex methods were also tested in our preliminary studies, but the results were almost equivalent (for the purpose of this work) but more time and resources consuming.

Unlike true online representations, skeletons from each signature are sets of unordered points. For instance, as in Figure 1, most offline signature skeletons can be regarded as sets of unordered pixels in a bitmap, even though some subsets of these pixels form segments that are clearly created in an unambiguous sequential hand gesture.

Comparisons between online signatures are straightforward, because points $\left(x_{O N L I N E}, y_{O N L I N E}\right)$ are ordered through time. Analogously, the comparison between two offline signatures may also be done through the comparison of sequences of points ( $\left.x_{O F F L I N E}, y_{O F F L I N E}\right)$, representing black pixel coordinates in skeletons. However, ambiguities concerning the ordering of points turns this task into a combinatorial optimization problem whose computational cost may be prohibitive.

To significantly reduce this cost, both methods proposed in this paper decompose offline signatures skeletons into Unambiguously Connected Skeleton Segments (UCSS), as illustrated in Figure 1. To define UCSS, an offline signature skeleton is regarded as connected graph $G(V, E)$ where the vertices $V$ are the points of the skeleton and the edges $E$ are bidirectional connection between neighbouring points (8connected neighbourhood). We also consider that the degree of a vertex $V$ is the number of neighbouring vertices that $V$ is connected to. Therefore, each UCSS is a sequence of directly connected vertices found between:

a) two vertices with degrees greater than 2 (internal segments),

b) an one-degree vertex and a vertex with degree greater than 2 (extremities), or

c) two one-degree vertices (isolated lines).

In other words, UCSS are specific sets of neighbouring points in a given skeleton. These points should form as long as possible connected sequences that unambiguously represent a single pen tip movement, a single direction, in spite of its sense. Nevertheless, it is not the purpose of this work to fully recover the trajectory of the signature, this is a much harder task that should be addressed in future works.

It should be noticed that, in general, the thinning process is noisy, what would demand the signature skeleton to be cleaned to guarantee 8-connected pixels. Fortunately, the thinning method used in this work is enough to guarantee connectivity 


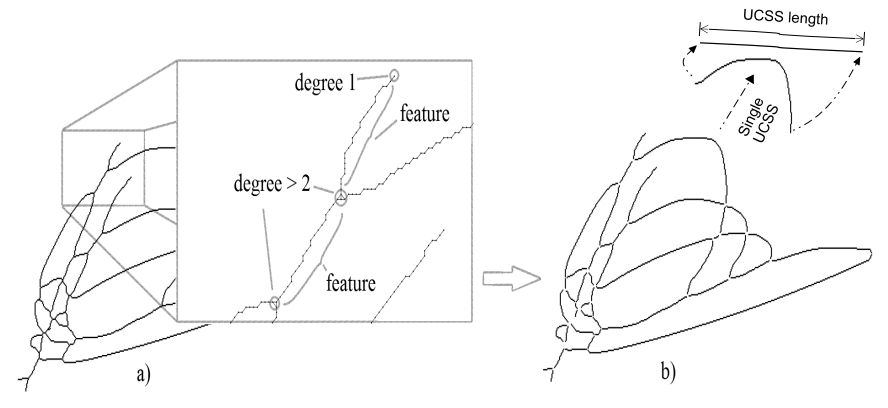

Fig. 1. (a) Inside the circles one finds the points of the skeleton that delimits UCSS. (b) Full skeleton as a set of extracted features(UCSS).

for UCSS extraction. In other words, this thinning process does not break lines, so the connectivity of the signature is maintained. Moreover, the 8-connected pixels are guaranteed except for those on image borders, because the image is a rectangular matrix of pixels.

We assume that each segment, $s_{m, n, k}$, is a portion of signature where points are unambiguously ordered, apart from a single ambiguity in the overall direction of the pen movement (i.e. one does not know in what end of the UCSS the movement of the pen begins). Thus, the $n$-th signature sample, $n=1,2, \ldots, N$, from the $m$-th signer, $m=1,2, \ldots, M$ is represented by a set $\left\{s_{m, n}\right\}=\left\{s_{m, n, 1}, s_{m, n, 2}, \ldots, s_{m, n, K}\right\}$, where $s_{m, n, k}$ is the $k$-th UCSS, or a sequence of $L_{m, n, k}$ pairs of coordinate points, $\left(x_{i}, y_{i}\right), 1<i \leq L_{m, n, k}$.

Moreover, to take into account the single ambiguity in the overall direction of the pen movement, each UCSS is represented twice: first with the sequence of pairs in a given order, $s_{m, n, k}=\left[\left(x_{1}, y_{1}\right),\left(x_{2}, y_{2}\right), \ldots,\left(x_{L_{m, n, k}}, y_{L_{m, n, k}}\right)\right]$, and then in reversed order, $s_{m, n, k}^{*}=$ $\left[\left(x_{L_{m, n, k}}, y_{L_{m, n, k}}\right),\left(x_{L_{m, n, k}-1}, y_{L_{m, n, k}-1}\right), \ldots,\left(x_{1}, y_{1}\right)\right]$.

\section{BASEline Method}

Two methods for automatic offline signature verification are proposed in this paper. The first method is considered as a baseline, for it is a straightforward application of Dynamic Time Warping to compute distances between UCSS. In this method, the standard DTW method under Itakura's restrictions [19] is applied to systematically compare every segment $s_{m, n, k}$ (and its reverse, $s_{m, n, k}^{*}$ ) to every segments of a given bag of segments, extracted from reference signatures. Consider, for instance, a test signature, $\left\{s_{m, t e s t}\right\}$ with $K_{\text {test }} \mathrm{UCSS}$, and a bag of segments, $\{B\}$, with $K_{B}$ UCSS (i.e. all segments, from all references, are merged into the single set $\{B\}$ ). Then, each UCSS in the test signature is compared to all $K_{B}$ UCSS from $\{B\}$, and the minimum distance is taken. In other words, each UCSS in a test signature is associated to the single UCSS in $\{B\}$ which yields the minimum DTW distance. More precisely,

$$
C_{m, t e s t}(k,\{B\})=\frac{1}{L_{m, t e s t, k}} \min _{j} D T W\left(s_{m, t e s t, k}, s_{B, j}\right),
$$

and the average distance between sets $\left\{s_{m, t e s t}\right\}$ and $\{B\}$ is given by

$$
\bar{C}_{m, \text { test }}(\{B\})=\frac{1}{K_{\text {test }}} \sum_{k=1}^{K_{\text {test }}} C_{m, t e s t}(k ;\{B\})
$$

where $\operatorname{DTW}\left(s_{m, t e s t, k}, s_{B, j}\right)$ stands for Dynamic Time Warping distance under Itakura's restriction between $s_{m, t e s t, k}$ and $s_{B, j}$, or its reversed version, $s_{B, j}^{*}$, depending on which one yields the lowest distance. Moreover, $k \in 1,2, \ldots, K_{\text {test }}, j \in 1,2, \ldots, K_{B}$, and test is a pointer to a signature from the test set. Moreover, $L_{m, t e s t, k}$ is the length (number of points) of the $k$-th UCSS, of the tested signature.

In this work, we assume that $N$ genuine signatures from a given individual are available as a reference set, denoted as $\left\{s_{m, r e f_{1}}\right\},\left\{s_{m, r e f_{2}}\right\}, \ldots,\left\{s_{m, r e f_{N}}\right\}$. Therefore, $\{B\}$ is the union of all $N$ references, namely: $\{B\}=$ $\left\{\left\{s_{m, r e f_{1}}\right\} \cup\left\{s_{m, r e f_{2}}\right\} \cup \ldots \cup\left\{s_{m, r e f_{N}}\right\}\right\}$, and $K_{B}$ is the resulting cardinality of $\{B\}$.

To provide a better score for tested signatures, we also define $N$ partial bags of segments, $\{B\}_{\text {ref }}$, where segments of the $i$-th reference signature are excluded from $\{B\}$. As a result, we are able to compute an average distance between each reference signature, $\left\{s_{m, r e f_{i}}\right\}$, and the corresponding remaining bag of segments, $\{B\}_{r e f_{i}}$, as follows

$$
\bar{C}_{0}(\{B\})=(1 / N) \sum_{i=1}^{N} \bar{C}_{m, r e f_{i}}\left(\{B\}_{r e f_{i}}\right) .
$$

Finally, the total distance between a tested signature $\left\{s_{m, t e s t}\right\}$ and a genuine set of references, summarised by the bag of segments $\{B\}$, is defined as:

$$
J_{m, r e f}=\frac{\bar{C}_{m, t e s t}(\{B\})}{\bar{C}_{0}(\{B\})},
$$

where $\bar{C}_{0}(\{B\})$ plays the role of a normalization factor.

An important drawback of this baseline method is the computation of more than $K_{B} \times K_{\text {test }}$ DTW distances in order to obtain a single score/cost for each tested signature, where $K_{B}$ is roughly $N$ times the average number of segments (UCSS) in a genuine signature. As a consequence, this method has a high computational burden.

\section{Proposed METhod WITH UCSS SUBSAMPLING}

To significantly alleviate the computational load of the baseline method, we encode each UCSS as an eight-dimensional (8D) vector that roughly represents the shape and the position of the encode UCSS, plus a scalar corresponding to the UCSS length (the length is given in terms of number of points in the UCSS), as illustrated in Figure 2.

This UCSS encoding strategy is the main aspect of the proposed method. We assume that almost all UCSS are short enough to prevent strong warping, therefore, one may get rid of the high DTW computation cost by replacing each UCSS with 4 subsampled points. In other words, it is assumed that UCSS comparisons through DTW are almost equivalent to the much faster Euclidean distance computation between the corresponding $8 \mathrm{D}$ vectors (UCSS length is not taken into 


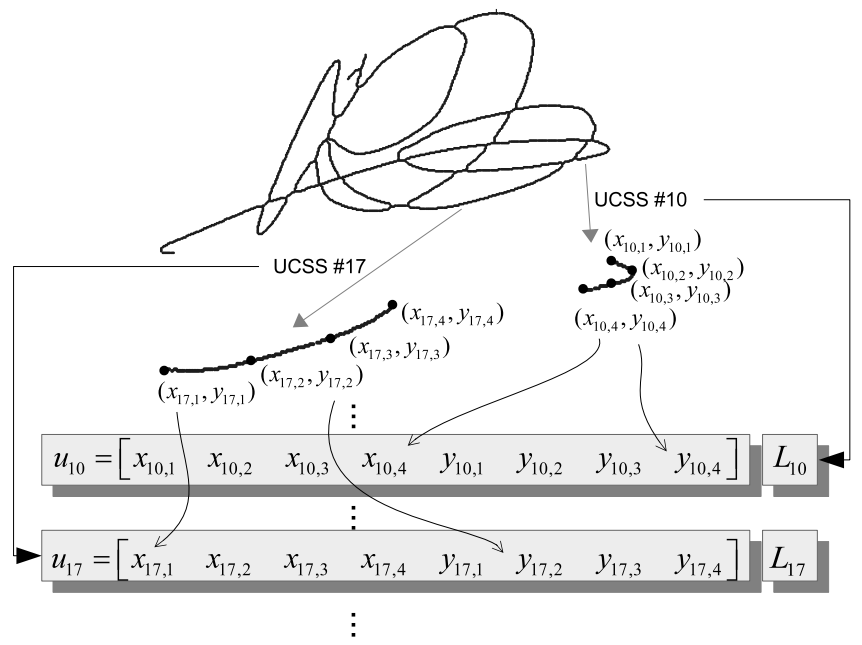

Fig. 2. Proposed method: illustration of $8 \mathrm{D}$ vectors and lengths of two segments (segments 10 and 17). Each segment (UCSS) is coded with $8+1=9$ scalars. The last scalar represents the segment length.

account when plain DTW is used). Indeed, a UCSS $s_{m, n, k}$ can be regarded as a composition of two sampled signals, say $x_{n}$ and $y_{n}$, with $n=1,2, \ldots, L_{U C S S}$, and the proposed coding scheme just takes 4 equally spaced subsamples of $x_{n}$ and $y_{n}$, thus yielding an 8-dimensional vector $u_{m, n, k}$. For practical purposes, we then assume that:

$$
\frac{\operatorname{DTW}\left(s_{m, n, i}, s_{m, n, j}\right)}{L_{m, n, i}} \approx \frac{D_{2}\left(u_{m, n, i}, u_{m, n, j}\right)}{4},
$$

where $D_{2}\left(u_{m, n, i}, u_{m, n, j}\right)$ is the accumulated squared Euclidean distance between the 4 corresponding points in the two compared UCSS. We highlight that, as for the definition of function $D T W$, in Equation 1, D2 is also defined as the minimum of two distances, where both $u_{m, n, j}$ and its reversed version, $u_{m, n, j}^{*}$ are considered. For the non-reversed $u_{m, n, j}$ only, we obtain $D_{2}\left(u_{m, n, i}, u_{m, n, j}\right)=\sum_{k=1}^{8}\left(u_{m, n, i}(k)-u_{m, n, j}(k)\right)^{2}$. Therefore, the comparison between two signatures is significantly simplified through the use of the following distance, as compared to that in Equation 1:

$$
C_{m, t e s t}(k,\{U\})=\frac{1}{4} \min _{j} D_{2}\left(u_{m, t e s t, k}, u_{U, j}\right),
$$

where $\{U\}$ is the set of all 4-points segments from the reference signatures. Analogously, every subset $\{B\}_{r e f_{i}}$ is replaced with $\{U\}_{r e f_{i}}$, and the final score/cost for a given signature can be computed as in Equation 2.

To obtain a single score/cost for each tested signature, the baseline method depends on the computation of about $K_{B} \times$ $K_{\text {test }}$ DTW distances, whose individual costs are quadratic with the average length of the UCSS. By contrast, the proposed method depends on the computation of the same amount $\left(K_{B} \times\right.$ $\left.K_{\text {test }}\right)$ of Euclidian distances between $4-\mathrm{D}$ vectors instead. For instance, if $K_{B}=150$ and $K_{\text {test }}=30$, and if UCSS are 58 points long on average (that is the actual average length for the databases used in this work) for a given signature, then the baseline method would demand about $150 \times 30 \times 58^{2}$ relevant floating point operations, to be compared with only $150 \times 30 \times 4$ for the proposed method.

\section{Databases and Experimental Protocol}

Experiments were first done with pseudo-offline versions of the MCYT-100 online signatures database, with 100 signatures, for which error rates (for online verification task) can be abundantly found in literature [6], [28], [17]. Afterwords, experiments we done again with the actual offline samples from the MCYT-75 database. These databases are subcorpus of the multimodal MCYT database [24]. For the MCYT-100 online, each writer provided 25 genuine signatures, whereas 5 different volunteers provided 25 skilled forgeries per signature. All signatures were acquired with a WACOM Intuos A6 USB Tablet at constant sampling rate of $100 \mathrm{~Hz}$. By contrast, for For the MCYT-75 offline, signatures were scanned and each writer provided 15 genuine samples. For each target subject, 15 skilled forgeries were provided.

Once features are available, signature authenticity verification can be performed. To simulate actual verification, most academic works randomly select a small number of genuine signature samples from each user (typically from 5 to 15) to play the role of a set of enrolled signatures. Then, samples from the remaining dataset of false and genuine signatures are randomly taken to simulate verification attempts. These test samples are compared to the enrolled samples, and a decision is made. If a genuine signature is rejected, it is called a false rejection error. By contrast, if a forgery is accepted, it is called a false acceptance error. Thus False Acceptance Rate (FAR) and False Rejection Rate (FRR), along with the operational point where FAR equals FRR, the Equal Error Rate (EER), are used in this work to compare method performances through four sets of experiments, namely:

E1: The MCYT-100 online database was converted into a pseudo-offline database, and methods for biometric verification were compared in terms of FAR, FRR and EER. To simulate an actual biometric system, $N$ genuine signatures were randomly chosen from the database to play the role of an enrolment card. Afterwards, $N$ other genuine signatures plus $N$ skilled forgeries of the same user were randomly sampled and compared with those into the enrolment card. Given a decision threshold, the proportion of true signatures whose costs were above this threshold (thus wrongly rejected as not genuine) was the estimated FRR, whereas the proportion of false signatures whose distances were below the threshold was the estimated FAR. In all experiments, $N$ was set to 5 genuine references, and the threshold was modified at small steps to yield Receiver Operating Characteristic (ROC) curves for each method.

To obtain the offline signatures from the MCYT-100 online database, we first convert each MCYT online sample into an image where only horizontal ( $\left.x_{O N L I N E}\right)$ and vertical $\left(y_{O N L I N E}\right)$ pen tip positions through time were considered, as follows:

(i) Points in each online signature were interpolated using splines to allow for oversampling of the otherwise sparse representation (due to the relatively low sampling rate of 100 samples per second). 
(ii) The oversampled set of points were numerically rounded to integer values, and

(iii) they were also dilated until segments were approximately four pixels wide.

These three steps were enough to convert the entire online data into offline signatures. Indeed, the resulting images were used in the Mean Opinion Score experiment detailed in Experiment E2.

E2: To quantify the human performance for the same task, we also prepared 239 cards, each one corresponding to a genuine source of signatures - a genuine signer - in the MCYT-100. These cards contained five genuine signatures on its left side, and ten signatures randomly chosen in its right side. Only 5 out of the 10 signatures on the right side were genuines, the other 5 were skilled forgeries, but volunteers taking part in the experiment were not informed of the proportion of true and skilled forgeries in the card. An example of these cards can be seen in Figure 3. These cards were presented to different students and lecturers in our university (willing volunteers), and these volunteers were carefully instructed to study the genuine signatures presented on the left part of the page, and then to label all signatures on the right part, by writing in the boxes next to each signature a $\mathrm{T}$, for true (genuine), or a $\mathrm{F}$, for false. No further a priori was provided (the volunteers did not know the proportions of true and false signatures in each card).

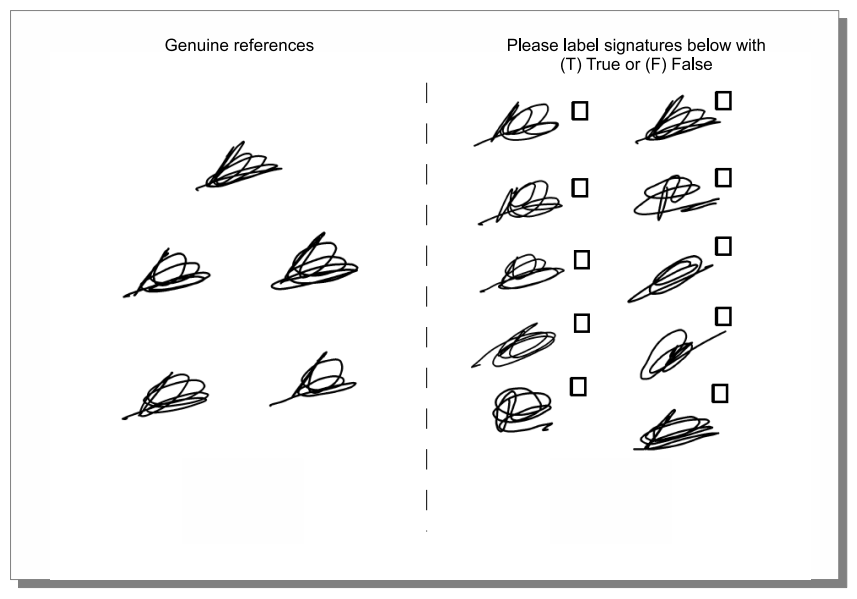

(a)

(b)

Fig. 3. (a) Reference panel with five randomly chosen genuine signatures (b) Panel with five randomly chosen forgeries and five randomly chosen genuine signatures.

E3: Each proposed method was repeated 50 times, under independent selection of genuine signatures and skilled forgeries. For each independent trial, the threshold decision was adjusted to yield the average EER for each method.

E4: The use of artificially obtained offline versions of online signatures, or pseudo-offline signatures, allows some interesting performance comparisons. However, the conversion of online signals into offline (bitmaps) can introduce relevant distortions. Thus, to test the consistency of results, further experiments were done with the true offline samples from the MCYT-75 database, under the same validation protocol.
In all experiments, either pseudo-offline signatures or actual offline ones were converted into pseudo-online representations, through the following steps:

(i) The standard skeletonization method described in [8] was applied to each signature image.

(ii) Resulting skeletons, or sets of 2D points from each signature sample were centered at the origin, whereas their variance in both vertical and horizontal directions were scaled to one.
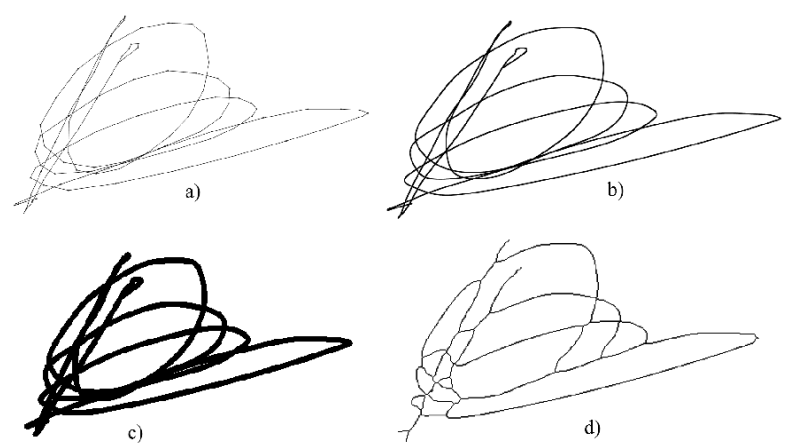

Fig. 4. (a) Online signature. (b) Oversampled online signature. (c) Dilated image - approximately four pixels wide lines. (d) Skeleton of the signature.

From the resulting pseudo-online versions of signatures, represented by a set of coordinate pairs, UCSS were extracted. It is noteworthy that the order in which these pairs are presented no longer stands for a discrete time counter, as in true online representation, for it now represents a mere skeleton point counter, whose correspondence to time ordering is unknown.

\section{RESULTS}

To provide a comparison scenario for the proposed methods based on UCSS, we included in E1 the method published in [29], which extracts projections of bitmaps corresponding to signatures, and compare them through a modified DTW, where so called stability measures are included to improve performances. We reproduced the best implementation of their method (as explained in their paper), and we applied it to the the same offline signatures we used to test our methods, under the same experimental protocol, with 5 genuine references, instead of 10, as in [29].

We also compared the computational burden of the two proposed methods to process and compare one enrolment card, with 5 genuine samples per signature, and to compute scores for all $20+25=45$ remaining signature samples per reference (respectively, $10+15=25$ remaining signature samples per reference in the MCYT-75 offline). The processing time for the baseline method was thousands times greater than that for the proposed method with UCSS subsampling. Surprisingly, the lighter method yielded a significantly better performance, as presented in Figure 5.

An interesting by-product of both methods is that after two UCSS are associated (according either to Equation 1 or to Equation 3), instead of comparing their shapes, one may just compare their lengths, thus yielding a new score corresponding 
TABLE I

RESULTS FOR EACH METHOD AFTER 50 INDEPENDENT RUNS WITH THE MCYT-100 ONLINE DATASET. EACH RUN CORRESPONDS TO A RANDOM PARTITION OF 5 REFERENCE AND 10 TEST SIGNATURES ( 5 GENUINE ONES).

\begin{tabular}{|c|c|c|}
\hline Method & EER & std. dev. \\
\hline I-Baseline & $32.2 \%$ & $1.4 \%$ \\
\hline II- Method by [29] & $36.7 \%$ & $0.6 \%$ \\
\hline III-Length based & $25.1 \%$ & $1.2 \%$ \\
\hline IV- Shape based (proposed) & $19.4 \%$ & $1.3 \%$ \\
\hline $\begin{array}{c}\text { V-Fusion (mean) of scores } \\
\text { from III and IV }\end{array}$ & $18.7 \%$ & $1.0 \%$ \\
\hline
\end{tabular}

to the absolute difference of associated lengths. This can be regarded as a third method, here referred to as the length based one, in Figure 5 and Tables I and II.

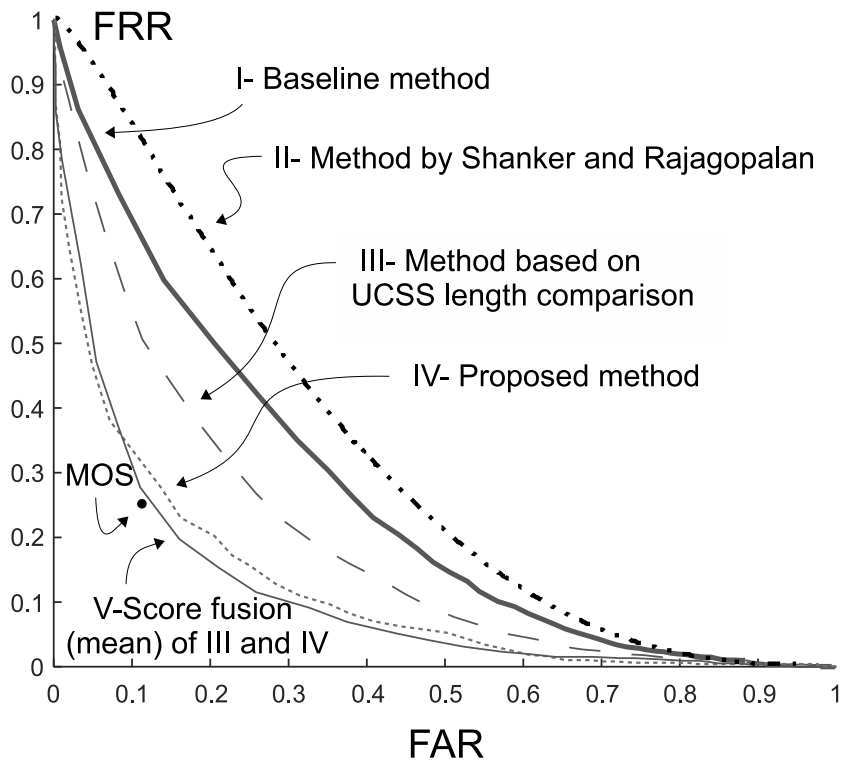

Fig. 5. ROC curves for all tested methods with the MCYT-100 online database. In this set of experiments, we use 5 randomly chosen reference signatures to simulate enrolment, and a pool of 20 remaining genuine signatures, along with 25 false ones to test the simulated system. A black dot also indicates the MOS performance for a subjective decision threshold that cannot be handled to yield a ROC.

Experiment E2 yielded a Mean Opinion Score (MOS) from 239 cards filled by 103 volunteers, and after comparing all provided labels to the true hidden labels, the estimated MOS was:

- $25.6 \%$ of False Rejection Rate and

- $11.0 \%$ of False Acceptance Rate.

This results corresponds to the dot in Figure 5, which allows the visual comparison between the rates FAR and FRR from MOS (to which the decision threshold cannot be known or handled) and the ROC curves from the automatic methods, for a range of possible decision thresholds.

Experiment $\mathbf{E 3}$ yield results presented in Table I, in terms of average EER and its standard deviation over 50 independent trials.

In all sets of experiments, scores of the methods III and IV are also fused through simple arithmetic mean, yielding an improved performance, as shown in Figure 5 and Tables I.
TABLE II

AVERAGE RESULTS FOR 50 INDEPENDENT RUNS WITH THE MCYT-75 OFFLINE DATASET. EACH RUN CORRESPONDS TO A RANDOM PARTITION OF 5 REFERENCE AND 10 TEST SIGNATURES ( 5 GENUINE ONES).

\begin{tabular}{|c|c|c|}
\hline Method & EER & std. dev. \\
\hline I-Baseline & $33.4 \%$ & $1.3 \%$ \\
\hline II- Method by [29] & $36.6 \%$ & $0.8 \%$ \\
\hline III-Length based & $25.7 \%$ & $1.6 \%$ \\
\hline IV- Shape based (proposed) & $20.9 \%$ & $1.3 \%$ \\
\hline $\begin{array}{c}\text { V-Fusion (mean) of scores } \\
\text { from III and IV }\end{array}$ & $20.1 \%$ & $1.2 \%$ \\
\hline
\end{tabular}

Results from Experiment $\mathbf{E 4}$ are presented in Table II. By comparing results in tables I and II, we observe that the pseudo-offline dataset from the MCYT-100 database have slightly better results than the true offline samples from the MCYT-75 database. This is expected because during the preprocessing steps the offline signatures are damaged in some areas, as can be seen in the small loops of the original signature in Figure 6. However, the similarities between the results, arising from the fact that the two bases are composed of exactly the same signatures extracted by different methods, make the pseudo-offline dataset, for the purpose of UCSS extraction, acceptable surrogates to the true samples from the MCYT-75 database.

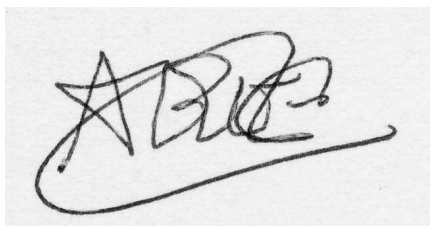

a)

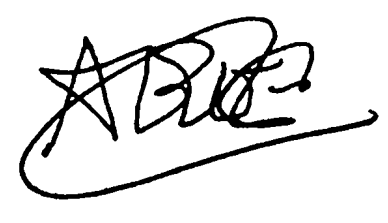

b)

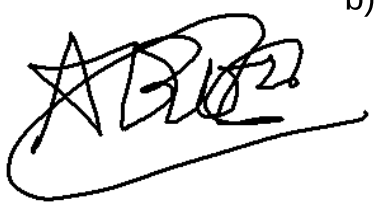

c)

Fig. 6. a) Original signature from the MCYT-75 database. b) Its preprocessed counterpart. c) Its pseudo-offline counterpart.

\section{DISCUSSION}

Results presented in tables I and II are consistent with human performances under the same protocol. Indeed, levels of accuracy reported in this work are not far from similar experiments with human presented in [21] and [5], for distinct datasets. However, state-of-the-art results for the MCYT-75 are significantly better, as presented in [32] and [18].

A common aspect found in these stete-of-the-art approaches is the use of genuine samples from other subjects enrolled in the system as negative examples (or random forgeries). This allows a kind of normalization score that significantly lower error rates. In [15], for instance, we can infer from Figure 4 (in [15]) that an EER of about $17 \%$ is obtained with the MCYT75 and 5 genuine signatures of reference, without negative 
examples. By contrast, it drops to about $10 \%$ when negative examples are taken into account.

Besides, a significant innovation found in [18] is that feature extraction is done automatically by a deep neural network (DNN). This approach is aligned to the new trend in pattern recognition, according to which hand-crafted feature extractors are replaced with automatic (learned by machines) ones. Impressive performances, far beyond what has been found in literature even for human benchmarks, seem to corroborate the effectiveness of this DNN based approach.

Indeed, by taking into account the human performance for MCYT-75, which is close to the results reported in this manuscript, but far worst than results reported in [18] for the same database, we may conjecture that volunteers were not attentive to signature details. This conjecture may explain why human performance, at least in our experiment, is close to what we obtained by discarding UCSS details.

On the other hand, a forensic specialist attentive to small ink deposition details would prefer a fine analysis of raw image patches, which is also retained by the parsimonious coding scheme proposed in [32], or by the convolutive patch processing of [18]. On the other hand, $\mathrm{CNN}$ based approaches, such as the one used in [18], face the challenge of explainability, which may be critical in sensitive applications such as forensics. Indeed, the parsimonious coding of patches found in [32] seems to be more prone to advances in explainability.

Similarly, and keeping the explainability issue in mind, we believe that it is possible to test, with the UCSS proposed in this work, if representing details is indeed a key aspect for attaining state-of-the-art performances. It can be done by the splitting of UCSS into smaller segments. But to also study the correspondence between human and machine performances, these new steps would be coupled with new MOS experiments, similar to E2, but with forensics specialists as volunteers. Clearly, it is a matter for future work.

Regarding the by-product method based on UCSS length, it is noteworthy that the use of either DTW or Euclidean distance to match UCSS is a necessary step. In other words, behind the apparent simplicity of this method, one should be aware that the matching of UCSS is a not so simple step of it, which also rises interesting questions. For instance, the superiority of the joint approach - where UCSS shape and length are combined - may have a connection to the lost signal of pen tip velocity, which in turn is the main signal for biometric verification in [3]. Indeed, UCSS shape (straightness) and length are expected to be somehow dependent on pen tip velocity, either through two-thirds power law, or through isochrony [31], and this dependency is also an attractive subject for further works. For now, we just conjecture that the fusion of length and shape based scores is somehow related to inferred velocity signal, given a signature image, which may explain its relatively good performance.

\section{CONCLUSION}

This brief work was inspired by the empirically observed superiority of online signature verification methods, as compared to most offline ones. Therefore all methods proposed here are based on skeletonization, possibly the most straightforward method to obtain pseudo-online signature representations from images. The baseline method cross-compares all unambiguously connected sequences of points from skeletons, where the conception of a Unambiguously Connected Skeleton Segment plays a pivotal role. By assuming that UCSS shapes and position are relevant information for biometric verification, we should expect that the systematic cross-comparison of UCSS, through DTW, would yield good performance, at a high computational cost.

However, it was noticed instead that the alternative method initially proposed to alleviate the high computational burden of the baseline method - by considering only 4 points per UCSS - yielded a significantly better performance, as compared to the baseline method. Moreover, even the very simple byproduct method based on the comparison between UCSS lengths performed better than the baseline method. Indeed, the baseline method only outperformed the method by [29], which uses an improved DTW, but relies upon marginal projections of signatures instead of segments like the UCSS proposed in this work.

From these results, we are tempted to conjecture that, on average, UCSS is a good segmentation option. Moreover, by taking into account our MOS experiment results, it also could be conjectured that UCSS shape details are not relevant for mimicking humans in biometric verification tasks, for results were improved when UCSS were down-sampled to 4 points only, approaching MOS based (non-expert) human performance. However, it contradicts the common sense that line quality details are important features for forensic experts in determining if a signature is a skilled forgery. We believe that this point is an interesting matter for future work, including MOS based experiments with forensic specialists.

\section{ACKNOWLEDGMENT}

This work was supported by grant from the Conselho Nacional de Desenvolvimento Científico e Tecnológico (CNPq) to J.M.

\section{REFERENCES}

[1] Y. M. Al-Omari, S. N. H. S. Abdullah, and K. Omar, "State-of-the-art in offline signature verification system," in Pattern Analysis and Intelligent Robotics (ICPAIR), 2011 International Conference on, vol. 1. IEEE, pp. 59-64, 2011, doi: 10.1109/ICPAIR.2011.5976912.

[2] L. Batista, D. Rivard, R. Sabourin, E. Granger, and P. Maupin, "State of the art in off-line signature verification," Pattern Recognition Technologies and Applications: Recent Advances, pp. 39-62, 2008, doi: 10.4018/9781-59904-807-9.ch003.

[3] J. Canuto, B. Dorizzi, J. Montalvão, and L. Matos, "On the infinite clipping of handwritten signatures," Pattern Recogn. Lett., vol. 79, no. C, pp. 38-43, Aug. 2016, doi: 10.1016/j.patrec.2016.04.026.

[4] J. Coetzer, B. M. Herbst, and J. A. du Preez, "Offline signature verification using the discrete radon transform and a hidden markov model," EURASIP Journal on Advances in Signal Processing, vol. 2004, no. 4, pp. 1-13, 2004, doi: 10.1155/S1110865704309042.

[5] J. Coetzer, B. Herbst, and J. Du Preez, "Off-line signature verification: a comparison between human and machine performance," in Tenth International Workshop on Frontiers in Handwriting Recognition. Suvisoft, 2006.

[6] M. A. Fierrer, J. F. Vargas, A. Morales, and A. Ordóñez, "Robustness of offline signature verification based on gray level features," IEEE Transactions on Information Forensics and Security, vol. 7, no. 3, pp. 966-977, 2012, doi: 10.1109/TIFS.2012.2190281. 
[7] M. A. Fierrer, J. F. Vargas, A. Morales, and A. Ordóñez, "An offline signature verification system based on fusion of local and global information," proceedings of the International Workshop on Biometric Authentication pp. 295-306, 2004, doi: 10.1007/978-3-540-25976-3_27.

[8] R. C. Gonzalez and R. E. Woods, Digital image processing, 2, 2007.

[9] V. Nguyen; M. Blumenstein "Techniques for static handwriting trajectory recovery: a survey" In: ACM. Proceedings of the 9th IAPR International Workshop on Document Analysis Systems. [S.l.], p. 463 - 470, 2010, doi: $10.1145 / 1815330.1815390$.

[10] K. Saeed, M. Tabedzki, M. Rybnik, and M. Adamski, "K3m: A universal algorithm for image skeletonization and a review of thinning techniques," Int. J. Appl. Math. Comput. Sci., vol. 20, no. 2, pp. 317 - 335, Jun. 2010, doi: 10.2478/v10006-010-0024-4.

[11] P. K. Saha,G. Borgefors, G. S. Baja. "A survey on skeletonization algorithms and their applications". Pattern Recognition Letters, Elsevier, v. 76, p. 3 - 12, 2016, doi: 10.1016/j.patrec.2015.04.006.

[12] A. Rosenfeld, "Axial representations of shape," Comput. Vis. Graph. Image Process., vol. 33, pp. 156 - 173, 1986, doi: 10.1016/0734189X(86)90113-1.

[13] R. W. Smith, "Computer processing of line images: A survey," Pattern Recognit., vol. 20, pp. 7 - 15, 1987, doi: 10.1016/0031-3203(87)90013-6.

[14] L. Lam, S. W. Lee, and C. Y. Suen, "Thinning methodologies - A comprehensive survey," IEEE Trans. Pattern Anal. Machine Intell., vol. 14, pp. 869 - 885, 1992, doi: 10.1109/34.161346.

[15] A. Gilperez, F. Alonso-Fernandez, S. Pecharroman, J. Fierrez, and J. Ortega-Garcia. "Off-line signature verification using contour features." In 11th International Conference on Frontiers in Handwriting Recognition, Canada, 2008.

[16] J. J. Zou, H. Yan, "Skeletonization of ribbon-like shapes based on regularity and singularity analyses". IEEE Transactions on Systems, Man, and Cybernetics, Part B (Cybernetics), IEEE, v. 31, n. 3, p. 401-407, 2001, doi: 10.1109/3477.931528.

[17] L. G. Hafemann, R. Sabourin, and L. S. Oliveira, "Offline handwritten signature verification-literature review," arXiv preprint arXiv:1507.07909, 2015 .

[18] L. G. Hafemann, R. Sabourin, and L. S. Oliveira, "Learning features for offline handwritten signature verification using deep convolutional neural networks." Pattern Recognition, vol. 70, pp. 163-176, 2017, 10.1016/j.patcog.2017.05.012.

[19] F. Itakura, "Minimum prediction residual principle applied to speech recognition," IEEE Transactions on Acoustics, Speech, and Signal Processing, vol. 23, no. 1, pp. 67-72, 1975, doi: 10.1109/TASSP.1975.1162641.

[20] S. Lee and J. C. Pan, "Offline tracing and representation of signatures," Systems, Man and Cybernetics, IEEE Transactions on, vol. 22, no. 4, pp. 755-771, 1992, doi: 10.1109/21.156588.

[21] D. Morocho, A. Morales, J. Fierrez, and R. Tolosana, "Signature recognition: establishing human baseline performance via crowdsourcing," in 2016 4th International Conference on Biometrics and Forensics (IWBF). IEEE, pp. 1-6, 2016, doi: 10.1109/IWBF.2016.7449680.

[22] E. Nel, J. A. du Preez, and B. M. Herbst, "Estimating the pen trajectories of multi-path static scripts using hidden markov models," in Document Analysis and Recognition, 2005. Proceedings. Eighth International Conference on. IEEE, pp. 41-45, 2005, doi: 10.1109/ICDAR.2005.106.

[23] V. Nguyen, M. Blumenstein, and G. Leedham, "Global features for the off-line signature verification problem," in 2009 10th International Conference on Document Analysis and Recognition. IEEE, pp. 13001304, 2009, doi: 10.1109/ICDAR.2009.123.

[24] J. Ortega-Garcia, J. Fierrez-Aguilar, D. Simon, J. Gonzalez, M. FaundezZanuy, V. Espinosa, A. Satue, I. Hernaez, J.-J. Igarza, C. Vivaracho et al., "Mcyt baseline corpus: a bimodal biometric database," IEE ProceedingsVision, Image and Signal Processing, vol. 150, no. 6, pp. 395-401, 2003, doi: 10.1049/ip-vis:20031078.

[25] R. Plamondon and S. N. Srihari, "Online and off-line handwriting recognition: a comprehensive survey," Pattern Analysis and Machine Intelligence, IEEE Transactions on, vol. 22, no. 1, pp. 63-84, 2000, doi: $10.1109 / 34.824821$

[26] Y. Qiao, M. Nishiara, and M. Yasuhara, "A framework toward restoration of writing order from single-stroked handwriting image," IEEE transactions on pattern analysis and machine intelligence, vol. 28, no. 11, pp. 1724-1737, 2006, doi: 10.1109/TPAMI.2006.216.

[27] Y. Qiao, J. Liu, and X. Tang, "Offline signature verification using online handwriting registration," CVPR'07. IEEE Conference on, pp. 1-8, 2007, doi: 10.1109/CVPR.2007.383263.

[28] A. S. Shah, M. Khan, F. Subhan, M. Fayaz, and A. Shah, "An offline signature verification technique using pixels intensity levels," International
Journal of Signal Processing, Image Processing and Pattern Recognition, vol. 9, no. 8, pp. 205-222, 2016, doi: 10.14257/ijsip.2016.9.8.18

[29] A. P. Shanker and A. Rajagopalan, "Off-line signature verification using dtw," Pattern recognition letters, vol. 28, no. 12, pp. 1407-1414, 2007, doi: 10.1016/j.patrec.2007.02.016.

[30] M. Diaz, M. A. Ferrer, A. Parziale and A. Marcelli. "Recovering Western On-line Signatures From Image-Based Specimens". 14th IAPR International Conference on Document Analysis and Recognition, Kyoto, Japan, 13-15 Nov. 2017, pp. 1 - 6, 2017, doi: 10.1109/ICDAR.2017.199.

[31] P. Viviani and T. Flash, "Minimum-jerk, two-thirds power law, and isochrony: converging approaches to movement planning.," Journal of Experimental Psychology: Human Perception and Performance, vol. 21, no. 1, pp. 32-53, 1995, doi: 10.1037/0096-1523.21.1.32.

[32] E. N. Zois, I. Theodorakopoulos, D. Tsourounis, and G. Economou. "Parsimonious Coding and Verification of Offline Handwritten Signatures.," CVPR workshop, 2017, doi: 10.1109/CVPRW.2017.92.

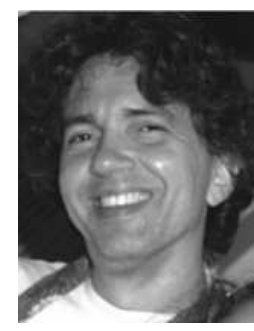

Jugurta Montalvão was born in Aracaju, Brazil, in 1968. He received the title of Electrical Engineer (1992) from the University of Campina Grande (UFPB II), Master in Electrical Engineering (1995) from the University of Campinas (UNICAMP) and Doctor in "Automatique et traitement du signal" (2000) from the University Paris-Sud XI. He joined the Department of Electrical Engineering of the Federal University of Sergipe (UFS) in 2005. His main research interests are: pattern recognition and signal processing.

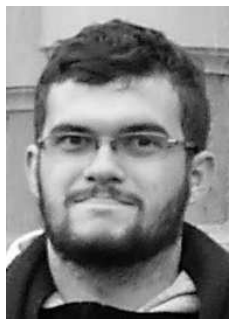

Luiz Miranda was born in Recife, Brazil, in 1991. $\mathrm{He}$ received the title of Electrical Engineer (2014) from the Federal University of Sergipe (UFS) and M.Sc. in Electrical Engineering (2017) from the Federal University of Sergipe (UFS). His main research interests are: pattern recognition and signal processing.

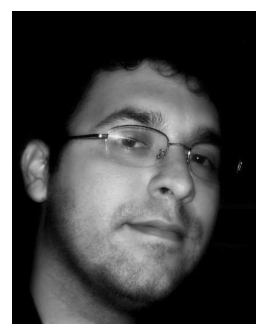

Jânio Canuto was born in Maceió, Brazil, in 1984. $\mathrm{He}$ received the title of Electrical Engineer (2007) from the Federal University of Sergipe (UFS), M.Sc. in Electrical Engineering (2010) from the State University of Campinas (UNICAMP) and Ph.D. in Computer Science (2014) from Télécom SudParis. $\mathrm{He}$ is currently a postdoc fellow at the Department of Computer Science of the Federal University of Sergipe (UFS). His main research interests are: pattern recognition and signal processing. 